\title{
Hypertoric Poisson homology in degree zero
}

\author{
Nicholas Proudfoot
}

\begin{abstract}
Etingof and Schedler formulated a conjecture about the degree zero Poisson homology of an affine cone that admits a projective symplectic resolution. We strengthen this conjecture in general and prove the strengthened version for hypertoric varieties. We also formulate an analogous conjecture for the degree zero Hochschild homology of a quantization of such a variety.
\end{abstract}

\section{Introduction}

Given a Poisson variety $\mathfrak{N}$ over $\mathbb{C}$, the degree zero Poisson homology group $\operatorname{HP}_{0}(\mathfrak{N})$ is defined to be the quotient of $\mathbb{C}[\mathfrak{N}]$ by the linear span of all brackets. If $\mathfrak{N}$ is affine and symplectic, then $\operatorname{HP}_{0}(\mathfrak{N})$ is isomorphic to $\mathrm{H}^{\operatorname{dim} \mathfrak{N}}(\mathfrak{N})$ via the map that takes the class of a function to the de Rham class of that function times the appropriate power of the symplectic form.

The next interesting case is when $\mathfrak{N}$ is an affine cone that admits a projective symplectic resolution $\mathfrak{M}$. In this case, we may deform the map $\mathfrak{M} \rightarrow \mathfrak{N}$ to a map $\mathscr{M} \rightarrow \mathscr{N}$, where $\mathscr{M}$ and $\mathscr{N}$ are varieties over the base $\mathrm{H}^{2}(\mathfrak{M})$ with zero fibers $\mathscr{M}_{0}=\mathfrak{M}$ and $\mathscr{N}_{0}=\mathfrak{N}$ [Nam11, Nam10]. Over a generic element $\lambda \in \mathrm{H}^{2}(\mathfrak{M})$, the map from $\mathscr{M}_{\lambda}$ to $\mathscr{N}_{\lambda}$ is an isomorphism of affine varieties. Then $\operatorname{HP}_{0}(\mathscr{N})$ is a module over $\mathbb{C}\left[\mathrm{H}^{2}(\mathfrak{M})\right]$ whose specialization at $\lambda$ is isomorphic to $\operatorname{HP}_{0}\left(\mathscr{N}_{\lambda}\right)$. When $\lambda$ is generic, this is isomorphic to $\mathrm{H}^{\operatorname{dim} \mathscr{N}_{\lambda}}\left(\mathscr{N}_{\lambda}\right) \cong \mathrm{H}^{\operatorname{dim} \mathscr{M}_{\lambda}}\left(\mathscr{M}_{\lambda}\right) \cong \mathrm{H}^{\operatorname{dim} \mathfrak{M}}(\mathfrak{M})$. Etingof and Schedler conjecture that the dimension of the zero fiber $\operatorname{HP}_{0}(\mathfrak{N})$ is also equal to that of $\mathrm{H}^{\operatorname{dim} \mathfrak{M}}(\mathfrak{M})$; equivalently, they conjecture that $\mathrm{HP}_{0}(\mathscr{N})$ is free over $\mathbb{C}\left[\mathrm{H}^{2}(\mathfrak{M})\right][\mathrm{ES} 11,1.3 .1(\mathrm{a})]$. They prove this conjecture for the Springer resolution and for Hilbert schemes of points on ALE spaces.

The goal of this paper is to both strengthen and prove this conjecture for hypertoric varieties, and to pose an analogous strengthening for other projective symplectic resolutions of affine cones. A hypertoric variety is an affine cone $\mathfrak{N}$ that admits a projective symplectic resolution $\mathfrak{M}$, equivariant for an effective Hamiltonian action of a torus $T$, with $\operatorname{dim} T=\frac{1}{2} \operatorname{dim} \mathfrak{N}$. A hypertoric variety $\mathfrak{N}$ comes with a "dual" hypertoric variety $\mathfrak{N}$ !, equipped with an action of its own torus $T$ !; the relationship between these dual pairs has been studied in [BLPW10] and [BLPW12]. One of the first properties of a dual pair is that the cohomology group $\mathrm{H}^{2}(\mathfrak{M})$ is canonically isomorphic to the Lie algebra of $T^{!}$. Our main result (Theorem 3.1) states that $\operatorname{HP}_{0}(\mathscr{N})$ is isomorphic as a graded module over $\mathbb{C}\left[\mathrm{H}^{2}(\mathfrak{M})\right] \cong \mathrm{H}^{*}\left(B T^{!}\right)$to the equivariant intersection cohomology group $\operatorname{IH}_{T^{!}}^{*}\left(\mathfrak{N}^{!}\right)$,

Received 16 May 2013, accepted in final form 17 December 2013.

2010 Mathematics Subject Classification 17B63

Keywords: Poisson homology, symplectic resolution, hypertoric

This journal is (C) Foundation Compositio Mathematica 2014. This article is distributed with Open Access under the terms of the Creative Commons Attribution Non-Commercial License, which permits non-commercial reuse, distribution, and reproduction in any medium, provided that the original work is properly cited. For commercial re-use, please contact the Foundation Compositio Mathematica.

The author is supported by NSF grant DMS-0950383. 


\section{Nicholas Proudfoot}

where the grading in Poisson homology is induced by the conical action of the multiplicative group. In particular, this implies that $\mathrm{HP}_{0}(\mathscr{N})$ is a free module over $\mathbb{C}\left[\mathrm{H}^{2}(\mathfrak{M})\right]$.

The relationship between a dual pair of hypertoric varieties is a special case of a relationship between pairs of projective symplectic resolutions called symplectic duality, which is being studied by Braden, Licata, Webster, and the author in a forthcoming paper; see [BLPW12, 1.5] for an overview of this notion. Examples of symplectic dual pairs, along with a conjectural extension of Theorem 3.1 to this setting (Conjecture 3.4), are given in Section 3.

We also note that Poisson homology of Poisson varieties is closely related to Hochschild homology of their quantizations. More precisely, let $A$ be a quantization of $\mathbb{C}[\mathscr{N}]$, and let $\operatorname{HH}_{0}(A)$ be the quotient of $A$ by the linear span of all commutators. Then $\operatorname{HH}_{0}(A)$ is a filtered vector space such that $\operatorname{gr} \mathrm{HH}_{0}(A)$ admits a canonical map from $\mathrm{HP}_{0}(\mathscr{N})$; Etingof and Schedler conjecture that this map is an isomorphism [ES11, 1.3.3]. In Conjecture 3.6, we conjecture the appropriate analogue of Theorem 3.1 and Conjecture 3.4 in the quantized setting.

The paper is organized as follows. In Section 2 we give a basic construction of a hypertoric variety and its dual. Section 3 is devoted to the statement of the main theorem and associated conjectures. We give a combinatorial presentation of $\operatorname{HP}_{0}(\mathscr{N})$ in Section 4 , which we use in Section 5 to prove that the main theorem holds on the numerical level (that is, we prove that the modules are isomorphic without obtaining a canonical isomorphism). Section 6 , which draws heavily on the machinery of [BP09], establishes the canonical isomorphism.

\section{Hypertoric varieties}

Fix a positive integer $n$, let $T^{n}:=\left(\mathbb{C}^{*}\right)^{n}$ be the coordinate torus, and let $X\left(T^{n}\right) \cong \mathbb{Z}^{n}$ be its character lattice. Let $\iota: G \hookrightarrow T^{n}$ be a connected algebraic subtorus, and let $\iota^{*}: X\left(T^{n}\right) \rightarrow X(G)$ be the pullback map on characters. We will impose two technical assumptions about this subtorus. First, we assume that the image of $\iota$ does not contain any of the one-dimensional coordinate subtori of $T^{n}$. Second, we assume that $\iota$ is unimodular, which means that for some (equivalently any) choice of basis for $X(G)$, all minors of $\iota^{*}$ belong to the set $\{-1,0,1\}$.

Let $V=\mathbb{C}^{n}$, equipped with the standard action of $T^{n}$. The vector space $V \oplus V^{*}$ carries a natural symplectic form, and the induced action of $T^{n}$ is Hamiltonian with moment map

$$
\Phi: V \oplus V^{*} \rightarrow X\left(T^{n}\right)_{\mathbb{C}} \cong \mathbb{C}^{n}
$$

given by the formula $\Phi(z, w)=\left(z_{1} w_{1}, \ldots, z_{n} w_{n}\right)$. The action of the subtorus $G$ on $V \oplus V^{*}$ is Hamiltonian with moment map

$$
\mu=\iota_{\mathbb{C}}^{*} \circ \Phi: V \oplus V^{*} \rightarrow X(G)_{\mathbb{C}} .
$$

Fix once and for all a generic character $\theta \in X(G)$. Let

$$
\mathscr{M}:=V \oplus V^{*} / \|_{\theta} G=\operatorname{Proj} \mathbb{C}\left[z_{1}, \ldots, z_{n}, w_{1}, \ldots, w_{n}, t\right]^{G}
$$

and

$$
\mathscr{N}:=V \oplus V^{*} / / 0 G=\operatorname{Spec} \mathbb{C}\left[z_{1}, \ldots, z_{n}, w_{1}, \ldots, w_{n}\right]^{G},
$$

where $G$ acts on $t$ with weight $\theta$. The map $\mu$ descends to a pair of maps

$$
\pi: \mathscr{M} \rightarrow X(G)_{\mathbb{C}} \quad \text { and } \quad \bar{\pi}: \mathscr{N} \rightarrow X(G)_{\mathbb{C}}
$$




\section{Hypertoric Poisson homology in Degree Zero}

and we let

$$
\mathfrak{M}:=\pi^{-1}(0) \quad \text { and } \quad \mathfrak{N}:=\bar{\pi}^{-1}(0) .
$$

These two spaces are called hypertoric varieties [BD00, Pro08]. The variety $\mathfrak{N}$ is an affine cone, and $\mathfrak{M}$ is a projective symplectic resolution of $\mathfrak{N}$.

Let $T:=T^{n} / G$; the action of $T^{n}$ on $V \oplus V^{*}$ descends to an effective Poisson action of $T$ on all of the aforementioned spaces. There is an additional (non-Poisson) action of $S \cong \mathbb{C}^{*}$ induced by the inverse scalar action on $V \oplus V^{*}$. We have the following $S \times T$-equivariant commutative diagram, where $T$ fixes $X(G)_{\mathbb{C}}$ and $S$ acts on $X(G)_{\mathbb{C}}$ with weight -2 :

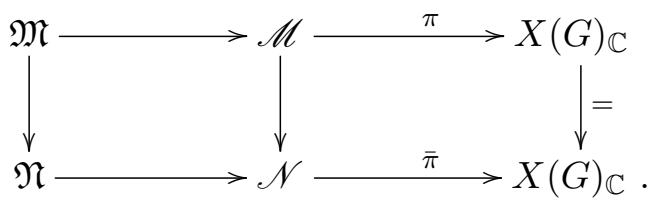

The vector space $X(G)_{\mathbb{C}}$ is isomorphic to $\mathrm{H}^{2}(\mathfrak{M})$ via the Kirwan map ${ }^{1}$, and $\mathscr{M}$ is the universal Poisson deformation of $\mathfrak{M}$ [Nam11, Nam10].

Remark 2.1. It may or may not be the case that $\mathscr{N}$ is the universal Poisson deformation of $\mathfrak{N}$. In general, this universal deformation is parameterized by $\mathrm{H}^{2}(\mathfrak{M}) / W$, where $W$ is the Namikawa Weyl group of $\mathfrak{N}$ (which may or may not be trivial). The deformation $\mathscr{N}$ is the pullback of the universal deformation from $\mathrm{H}^{2}(\mathfrak{M}) / W$ to $X(G)_{\mathbb{C}}$. We will refer to this as the quasi-universal deformation of $\mathfrak{N}$.

Let $\left(T^{n}\right)^{*}$ be the dual torus to $T^{n}$, and let $G^{!} \subset\left(T^{n}\right)^{*}$ be the inclusion of the connected subtorus whose Lie algebra is perpendicular to that of $G \subset T^{n}$. Fixing a generic character $\theta^{!} \in X\left(G^{!}\right)$, we may construct new spaces

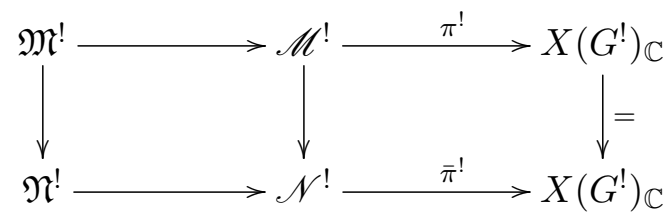

as above. This diagram will be $S \times T^{!}$-equivariant, where $S$ is as before and $T^{!}:=\left(T^{n}\right)^{*} / G^{!}$. The relationship between $\mathfrak{N}$ and $\mathfrak{N}^{!}$(and all of their associated geometry and representation theory) was explored in detail in [BLPW10] and [BLPW12].

\section{Results and conjectures}

For any $\lambda \in X(G)_{\mathbb{C}} \cong \mathfrak{g}^{*}$, let

$$
\mathscr{M}_{\lambda}:=\pi^{-1}(\lambda) \text { and } \mathscr{N}_{\lambda}:=\bar{\pi}^{-1}(\lambda) .
$$

The degree zero Poisson homology group $\operatorname{HP}_{0}(\mathscr{N})$ is a module over $\mathbb{C}\left[X(G)_{\mathbb{C}}\right] \cong$ Sym $\mathfrak{g}$, and for any $\lambda$, we have

$$
\operatorname{HP}_{0}\left(\mathscr{N}_{\lambda}\right) \cong \operatorname{HP}_{0}(\mathscr{N}) \otimes_{\text {Sym g }} \mathbb{C}_{\lambda}
$$

The action of $S$ induces positive integer gradings of $\operatorname{HP}_{0}(\mathscr{M})$ and Sym $\mathfrak{g}$, with $\mathfrak{g}$ sitting in degree two. This grading descends to $\operatorname{HP}_{0}(\mathfrak{N})=\operatorname{HP}_{0}\left(\mathscr{N}_{0}\right)$, but not to $\operatorname{HP}_{0}\left(\mathscr{N}_{\lambda}\right)$ for nonzero $\lambda$.

\footnotetext{
${ }^{1}$ The fact that the Kirwan map is an isomorphism in degree 2 follows from the assumption that the image of $\iota$ contains no coordinate subtorus of $T^{n}$.
} 


\section{Nicholas Proudfoot}

Observe that $T^{!}$is canonically dual to $G$; in particular, $\mathrm{H}^{*}\left(B T^{!}\right)$is canonically isomorphic as a graded ring to Sym $\mathfrak{g}$. Our main result is the following.

Theorem 3.1. There is a canonical isomorphism of graded Sym $\mathfrak{g}$-modules $\operatorname{HP}_{0}(\mathscr{N}) \cong \mathrm{IH}_{T^{!}}^{*}\left(\mathfrak{N}^{!}\right)$. In particular, $\operatorname{HP}_{0}(\mathfrak{N})$ is isomorphic as a graded vector space to $\operatorname{IH}^{*}\left(\mathfrak{N}^{!}\right)$.

Remark 3.2. A combinatorial interpretation of the intersection cohomology Betti numbers of $\mathfrak{N}^{\text {! }}$ was given in [PW07, 4.3]. In particular, they vanish in odd degree, thus $\mathrm{IH}_{T^{!}}^{*}\left(\mathfrak{N}^{!}\right)$is a free $\operatorname{Sym} \mathfrak{g}$ module. Theorem 3.1 is therefore a strengthening in the hypertoric case of a conjecture of Etingof and Schedler [ES11, 1.3.1(a)], which says (for an affine cone that admits a projective symplectic resolution) that the degree zero Poisson homology of the quasi-universal deformation is a free module over the coordinate ring of the base. In fact, proving freeness (Corollary 5.3) is one of the steps toward proving Theorem 3.1.

Remark 3.3. In subsequent work with Schedler, we generalize Theorem 3.1 by computing the higher Poisson-de Rham homology groups of hypertoric varieties. We do this by proving Conjectures 1.3.1(b) and 1.3.1(c) of [ES11] for hypertoric varieties, which state roughly that the higher Poissonde Rham homology groups of $\mathfrak{N}$ may be understood in terms of the degree zero Poisson homology groups of the slices to the various symplectic leaves of $\mathfrak{N}$. Since these slices are themselves hypertoric varieties [PW07, 2.4], this allows us to leverage Theorem 3.1 to obtain a description of all of the higher groups.

The relationship between $\mathfrak{N}$ and $\mathfrak{N}^{!}$is a special case of a phenomenon called symplectic duality [BLPW12, 1.5], which relates pairs of affine cones that admit projective symplectic resolutions. Theorem 3.1 invites the following generalization.

ConjeCture 3.4. Let $\mathfrak{N}$ and $\mathfrak{N}^{\prime}$ be a symplectic dual pair of cones admitting projective symplectic resolutions $\mathfrak{M}$ and $\mathfrak{M}^{!}$. Let $\mathscr{N}$ be the quasi-universal deformation of $\mathfrak{N}$, and let $T^{!}$be a maximal torus in the Hamiltonian automorphism group of $\mathfrak{M}^{!}$. There exist isomorphisms of graded vector spaces

$$
\mathrm{HP}_{0}(\mathfrak{N}) \cong \mathrm{IH}^{*}\left(\mathfrak{N}^{!}\right) \text {and } \operatorname{HP}_{0}(\mathscr{N}) \cong \mathrm{IH}_{T^{!}}^{*}\left(\mathfrak{N}^{!}\right)
$$

where the second isomorphism is compatible with the module structure over $\mathbb{C}\left[\mathrm{H}^{2}(\mathfrak{M})\right] \cong \mathrm{H}^{*}\left(B T^{!}\right)$.

Note that $\mathrm{IH}^{*}\left(\mathfrak{N}^{!}\right) \subset \mathrm{H}^{*}\left(\mathfrak{M}^{!}\right)$is always concentrated in even degree [BPW12, 2.5], and therefore the deformation coming from equivariant cohomology is always free. Thus Conjecture 3.4 would imply the conjecture of Etingof and Schedler from Remark 3.2 for any cone that has a symplectic dual.

A general definition of a symplectic dual pair appears in a forthcoming paper by Braden, Licata, Webster, and the author. In addition to the hypertoric examples discussed here, other examples include the following.

- If $G$ is a simple algebraic group and $G^{L}$ is its Langlands dual, then the nilpotent cone of $\mathfrak{g}$ should be dual to the nilpotent cone of $\mathfrak{g}^{L}$. In this case, both Poisson homology and intersection cohomology are one-dimensional [ES10, 1.6].

- A normal slice inside the nilpotent cone to a subregular nilpotent orbit in a simply-laced simple Lie algebra $\mathfrak{g}$ should be dual to the closure of the minimal nontrivial nilpotent orbit in $\mathfrak{g}^{L} \cong \mathfrak{g}$. The Poisson homology Poincaré polynomial of the slice is computed in [AL98] and the intersection cohomology Poincaré polynomial of the orbit closure is computed in [MOV05, 6.4.2], and they agree. ${ }^{2}$

\footnotetext{
${ }^{2}$ In the arXiv version of [MOV05], it is 6.2.2.
} 


\section{Hypertoric Poisson homology in DEGReE Zero}

- Let $X(r, n)$ be the symmetric scheme of $n$ points on the quotient of $\mathbb{C}^{2}$ by the symplectic action of $\mathbb{Z} / r \mathbb{Z}$. Let $Y(r, n)$ be the Uhlenbeck space which is obtained by taking the spectrum of the ring of global functions on the moduli space of framed torsion-free sheaves on $\mathbb{P}^{2}$ of rank $r$ and charge $n$. Then $X(r, n)$ is dual to $Y(r, n)$. The Poisson homology Poincaré polynomial of $X(r, n)$ is computed in [ES12, Eq. (1.1.15)] and the intersection cohomology Poincaré polynomial of $Y(r, n)$ can be deduced from [Nak99, 5.15(2)] and [Bar00, 3.7], and they agree.

Remark 3.5. Note that we do not include the word "canonical" in Conjecture 3.4. Though the isomorphism is canonical in the hypertoric case, the proof of this fact uses techniques that are very specific to hypertoric varieties.

Let $A$ be a quantization of $\mathscr{N}$, that is, a filtered algebra whose associated graded ring is isomorphic to $\mathbb{C}[\mathscr{N}]$ (with grading induced by the $S$-action), inducing the given Poisson structure. Then $A$ has a central quotient $A_{0}$ which is a quantization of $\mathfrak{N}$. The Hochschild homology group $\mathrm{HH}_{0}(A):=A /[A, A]$ admits a filtration whose associated graded module admits a surjection from $\mathrm{HP}_{0}(\mathscr{N})$ as a graded module over $Z(A) \cong \mathrm{Sym} \mathfrak{g}$; in particular, we also get a surjection from $\mathrm{HP}_{0}(\mathfrak{N})$ to $\mathrm{HH}_{0}\left(A_{0}\right)$. If the conjecture [ES11, 1.3.1(a)] holds, then these surjections are both isomorphisms [ES11, $\S 1.3]$. The appropriate analogue of Conjecture 3.4 is the following.

Conjecture 3.6. In the situation of Conjecture 3.4, there exist isomorphisms of filtered vector spaces

$$
\operatorname{HH}_{0}\left(A_{0}\right) \cong \operatorname{IH}_{S}^{*}\left(\mathfrak{N}^{!}\right) \otimes_{\mathbb{C}[u]} \mathbb{C}_{1} \text { and } \operatorname{HH}_{0}(A) \cong \operatorname{IH}_{S \times T^{!}}^{*}\left(\mathfrak{N}^{!}\right) \otimes_{\mathbb{C}[u]} \mathbb{C}_{1},
$$

where $\mathbb{C}_{1}$ is the one-dimensional module over $\mathrm{H}^{*}(B S) \cong \mathbb{C}[u]$ annihilated by $u-1$. The second isomorphism is compatible with the module structure over $Z(A) \cong \mathrm{H}^{*}\left(B T^{!}\right)$.

In the hypertoric case, we may take $A$ to be the ring of $G$-invariant differential operators on $V$, which is called the hypertoric enveloping algebra [BLPW12, 5.2]. Since we know by Theorem 3.1 that $\operatorname{HP}_{0}(\mathscr{N})$ is a free module, $\mathrm{HH}_{0}(A)$ must be a filtered free module whose associated graded module is canonically isomorphic to $\mathrm{HP}_{0}(\mathscr{N})$. In this case, we expect the second isomorphism of Conjecture 3.6 to be canonical, and it should be possible to establish this isomorphism using techniques similar to those that we use to prove Theorem 3.1. The main task would be to extend the results of [BP09] to the $S$-equivariant setting, which would be straightforward.

Remark 3.7. Let $\lambda \in X(G)_{\mathbb{C}}$ be generic. In this case $\mathscr{N}_{\lambda}$ is smooth and affine, thus

$$
\operatorname{HP}_{0}(\mathscr{N}) \otimes_{\operatorname{Sym} \mathfrak{g}} \mathbb{C}_{\lambda} \cong \operatorname{HP}_{0}\left(\mathscr{N}_{\lambda}\right) \cong \mathrm{H}^{\operatorname{dim} \mathscr{N}_{\lambda}}\left(\mathscr{N}_{\lambda}\right) \cong \mathrm{H}^{\operatorname{dim} \mathscr{M}_{\lambda}}\left(\mathscr{M}_{\lambda}\right) \cong \mathrm{H}^{\operatorname{dim} \mathfrak{M}}(\mathfrak{M})
$$

Here the second isomorphism is given by multiplication by the appropriate power of the symplectic form, the third isomorphism comes from the fact that $\mathscr{N}_{\lambda}$ is isomorphic to $\mathscr{M}_{\lambda}$, and the fourth isomorphism comes from the topological triviality of the family $\pi: \mathscr{M} \rightarrow X(G)_{\mathbb{C}}$. On the other hand, [BLPW12, 7.21] implies that $\operatorname{IH}_{T^{!}}^{*}\left(\mathfrak{N}^{!}\right) \otimes_{\operatorname{Symg}} \mathbb{C}_{\lambda}$ is canonically dual to $\mathrm{H}^{\operatorname{dim} \mathfrak{M}}(\mathfrak{M})$. Comparing these two results gives us a nondegenerate bilinear form on the vector space $\mathrm{H}^{\operatorname{dim} \mathfrak{M}}(\mathfrak{M})$ that depends nontrivially on $\lambda$. It would be interesting to understand this family of bilinear forms in more detail (see Example 3.8 for the first interesting case).

An analogue of [BLPW12, 7.21] is part of the package for all symplectic dual pairs, so a similar phenomenon should arise for all of the examples mentioned above.

Example 3.8. Suppose that $n=3$ and $G \subset T^{3}$ is the determinant 1 subtorus. In this case, $\mathfrak{N}$ is the Kleinian singularity of type $A_{2}$ and $\mathfrak{M}$ is its minimal resolution. We may identify the groups 


\section{Nicholas Proudfoot}

$X(G)_{\mathbb{C}} \cong H^{2}(\mathfrak{M})$ with $\mathbb{C}^{2}$ by taking the two projective lines in the exceptional fiber as a basis for $H_{2}(\mathfrak{M})$. For $u, v \in \mathbb{C}^{2} \cong H^{2}(\mathfrak{M})$ and $\lambda \in \mathbb{C}^{2} \cong X(G)_{\mathbb{C}}$, we consider the pairing of $u$ with $v$ using the bilinear form defined by $\lambda$ via Remark 3.7. This is well defined if and only if $\lambda$ is generic enough for $\mathscr{N}_{\lambda}$ to be affine, which in this case means that $\lambda_{1}, \lambda_{2}$, and $\lambda_{1}+\lambda_{2}$ are all nonzero. Explicit computation then yields

$$
(u, v)_{\lambda}=\frac{1}{\left[\lambda_{1} \lambda_{2}\left(\lambda_{1}+\lambda_{2}\right)\right]^{2}}\left(\lambda_{2}^{2}\left(2 \lambda_{1}+\lambda_{2}\right) u_{1} v_{1}+\lambda_{1} \lambda_{2}^{2} u_{1} v_{2}-\lambda_{1}^{2} \lambda_{2} u_{2} v_{1}-\lambda_{1}^{2}\left(2 \lambda_{2}+\lambda_{1}\right) u_{2} v_{2}\right) .
$$

In general, the family of bilinear forms will be homogeneous in $\lambda$ of degree $-n$.

\section{A presentation of $\operatorname{HP}_{0}(\mathscr{N})$}

For each $\alpha \in X\left(T^{n}\right) \subset\left(\mathfrak{t}^{n}\right)^{*}$, consider the differential operator

$$
\partial_{\alpha}: \operatorname{Sym} \mathfrak{t}^{n} \rightarrow \operatorname{Sym}^{n}
$$

defined by putting $\partial_{\alpha} x=\langle\alpha, x\rangle$ for all $x \in \mathfrak{t}^{n}$ and extending via the Leibniz rule. It will be convenient for us to work in coordinates; we identify $X\left(T^{n}\right)$ with $\mathbb{Z}^{n}$ and Sym $\mathfrak{t}^{n}$ with $\mathbb{C}\left[e_{1}, \ldots, e_{n}\right]$, and we have $\partial_{\alpha} e_{i}=\alpha_{i}$ (the $i^{\text {th }}$ coordinate of $\alpha \in \mathbb{Z}^{n}$ ). We will be particularly interested in those operators $\partial_{\alpha}$ for which $\alpha$ is in the kernel of $\iota^{*}: X\left(T^{n}\right) \rightarrow X(G)$.

Consider the vector space

$$
J:=\mathbb{C}\left\{\partial_{\alpha} e^{\beta} \mid \alpha \in \operatorname{ker} \iota^{*}, \beta \in \mathbb{N}^{n}, \operatorname{Supp}(\alpha) \subset \operatorname{Supp}(\beta)\right\} \subset \mathbb{C}\left[e_{1}, \ldots, e_{n}\right],
$$

where the support of an element of $\alpha \in \mathbb{Z}^{n}$ is the set of $i$ such that $\alpha_{i} \neq 0$. This is not an ideal, but it is a module over

$$
\operatorname{Sym} \mathfrak{g} \subset \operatorname{Sym}^{n}=\mathbb{C}\left[e_{1}, \ldots, e_{n}\right]
$$

because $\partial_{\alpha} x=0$ for all $x \in \mathfrak{g} \subset \mathfrak{t}^{n}$ and $\alpha \in \operatorname{ker} \iota^{*}$.

Remark 4.1. For any $\alpha$ and $\beta$, we have $\partial_{\alpha}+\partial_{\beta}=\partial_{\alpha+\beta}$. For this reason, we may restrict our attention in the definition of $J$ to those $\alpha$ which are primitive and have minimal support. The unimodularity condition implies that for such an $\alpha, \alpha_{i} \in\{-1,0,1\}$ for all $i$. Such an $\alpha$ is called a signed circuit, and there are only finitely many of them.

Proposition 4.2. The homology group $\operatorname{HP}_{0}(\mathscr{N})$ is isomorphic to $\mathbb{C}\left[e_{1}, \ldots, e_{n}\right] / J$ as a graded Sym $\mathfrak{g}$-module.

Proof. Recall that $\operatorname{HP}_{0}(\mathscr{N})$ is defined as the quotient of $\mathbb{C}[\mathscr{N}]=\mathbb{C}\left[z_{1}, \ldots, z_{n}, w_{1}, \ldots, w_{n}\right]^{G}$ by the linear span of all brackets, and consider the graded Sym $\mathfrak{g}$-module homomorphism

$$
\psi: \mathbb{C}\left[e_{1}, \ldots, e_{n}\right] \rightarrow \operatorname{HP}_{0}(\mathscr{N})
$$

taking $e_{i}$ to the class represented by $z_{i} w_{i}$. We will show that $\psi$ is surjective with kernel $J$.

The invariant ring $\mathbb{C}\left[z_{1}, \ldots, z_{n}, w_{1}, \ldots, w_{n}\right]^{G}$ consists of all monomials $z^{\beta} w^{\delta}$ with $\beta, \delta \in \mathbb{N}^{n}$ and $\iota^{*}(\beta-\delta)=0$. The Poisson bracket is given by the formula

$$
\{p(z, w), q(z, w)\}=\sum_{i=1}^{n} r_{i}(z, w),
$$

where $r_{i}(z, w)$ is the coefficient of $d z_{i} \wedge d w_{i}$ in the expansion of $d p(z, w) \wedge d q(z, w)$. In particular, we have

$$
\left\{z_{i} w_{i}, z^{\beta} w^{\delta}\right\}=\left(\delta_{i}-\beta_{i}\right) z^{\beta} w^{\delta}
$$




\section{Hypertoric Poisson homology in DEGReE Zero}

This tells us that the class of $z^{\beta} w^{\delta}$ in $\operatorname{HP}_{0}(\mathscr{N})$ is zero unless $\beta=\delta$, and therefore that $\psi$ is surjective.

The remaining relations in $\operatorname{HP}_{0}(\mathscr{N})$ come from brackets of the form

$$
\left\{(z w)^{\gamma} z^{\beta} w^{\delta},(z w)^{\epsilon} z^{\delta} w^{\beta}\right\}
$$

for some $\beta, \delta, \gamma, \epsilon \in \mathbb{N}^{n}$ with $\iota^{*}(\beta-\delta)=0$. This bracket expands to

$$
\sum_{i=1}^{n}\left(\beta_{i}-\delta_{i}\right)\left(\beta_{i}+\delta_{i}+\gamma_{i}+\epsilon_{i}\right)(z w)^{\beta+\delta+\gamma+\epsilon} /\left(z_{i} w_{i}\right)=\psi\left(\partial_{\beta-\delta} e^{\beta+\delta+\gamma+\epsilon}\right) .
$$

Thus the kernel of $\psi$ is contained in $J$.

The fact that $J$ is contained in the kernel follows from unimodularity: given a signed circuit $\alpha$, we can find a unique pair $\beta, \delta \in \mathbb{N}^{n}$ such that $\alpha=\beta-\delta$ and $\operatorname{Supp}(\alpha)=\operatorname{Supp}(\beta) \sqcup \operatorname{Supp}(\delta)$. Then every monomial whose support contains that of $\alpha$ is a multiple of $e^{\beta+\delta}$.

Remark 4.3. If the condition of unimodularity is dropped, then the last sentence of the proof of Proposition 4.2 will fail, and this will cause Theorem 3.1 to fail, as well. Geometrically, unimodularity ensures that $\mathfrak{M}$ is a manifold rather than an orbifold. Thus Conjecture 3.4 is really about affine cones that admit symplectic resolutions; orbifold resolutions are not good enough.

\section{Numerics}

In this section we will prove Theorem 3.1 minus the word "canonical". That is, we will show that $\operatorname{HP}_{0}(\mathscr{N})$ is a free Sym $\mathfrak{g}$-module with the same Hilbert series as $\mathrm{IH}_{T^{!}}^{*}\left(\mathfrak{N}^{!}\right)$. This will be a necessary first step toward establishing the canonical isomorphism, which we will do in the next section.

Lemma 5.1. The graded Sym $\mathfrak{g}$-module $\mathbb{C}\left[e_{1}, \ldots, e_{n}\right] / J$ degenerates flatly to $\mathbb{C}\left[e_{1}, \ldots, e_{n}\right] / J_{\Delta^{\mathrm{bc}}}$, where $J_{\Delta}$ bc is the Stanley-Reisner ideal of the broken circuit complex of the matroid associated with the inclusion $G \subset T^{n}$.

Proof. Consider the graded lexicographical term order on $\mathbb{C}\left[e_{1}, \ldots, e_{n}\right]$, which allows us to define the initial Sym $\mathfrak{g}$-module

$$
\operatorname{in}(J):=\{\operatorname{in}(f) \mid f \in J\} \subset \mathbb{C}\left[e_{1}, \ldots, e_{n}\right] .
$$

We want to show that $\operatorname{in}(J)=J_{\Delta^{\text {bc }}}$. For all $\alpha \in \operatorname{ker} \iota^{*}$ and $\beta \in \mathbb{N}^{n}$ with $\operatorname{Supp}(\alpha) \subset \operatorname{Supp}(\beta)$, $\operatorname{in}\left(\partial_{\alpha} e^{\beta}\right)=\alpha_{i} \beta_{i} e^{\beta} / e_{i}$, where $i$ is the maximal element of the support of $\alpha$. Thus we have

$$
\operatorname{in}(J) \supset \mathbb{C}\left\{\operatorname{in}\left(\partial_{\alpha} e^{\beta}\right) \mid \alpha \in \operatorname{ker} \iota^{*}, \beta \in \mathbb{N}^{n}, \operatorname{Supp}(\alpha) \subset \operatorname{Supp}(\beta)\right\}=J_{\Delta^{\mathrm{bc}}} .
$$

We do not yet know whether or not this containment is an equality, because the set of initial terms of a basis for a module need not form a basis for the initial module. What we do know is that $\mathbb{C}\left[e_{1}, \ldots, e_{n}\right] / \operatorname{in}(J)$ is isomorphic to a quotient of $\mathbb{C}\left[e_{1}, \ldots, e_{n}\right] / J_{\Delta}$ bc by some Sym $\mathfrak{g}$-submodule, which we will call $Q$.

By [PW07, 4.3] and [PS06, Proposition 1], $\mathbb{C}\left[e_{1}, \ldots, e_{n}\right] / J_{\Delta^{\mathrm{bc}}}$ is a free Sym $\mathfrak{g}$-module with the same Hilbert series as $\mathrm{IH}_{T^{!}}^{*}\left(\mathfrak{N}^{!}\right)$. As noted in Remark 3.7 , for generic $\lambda \in X(G)_{\mathbb{C}} \cong \mathfrak{g}^{*}$, $\operatorname{HP}_{0}(\mathscr{N}) \otimes_{\text {Sym g }} \mathbb{C}_{\lambda}$ is isomorphic to $\mathrm{H}^{\operatorname{dim} \mathfrak{M}}(\mathfrak{M})$, which is in turn dual to $\operatorname{IH}_{T^{!}}^{*}\left(\mathfrak{N}^{!}\right) \otimes_{\text {Sym } \mathfrak{g}} \mathbb{C}_{\lambda}$. In particular $\operatorname{HP}_{0}(\mathscr{N}) \otimes_{S y m g} \mathbb{C}_{\lambda}$ and $\operatorname{IH}_{T^{!}}^{*}\left(\mathfrak{N}^{!}\right) \otimes_{S y m g} \mathbb{C}_{\lambda}$ have the same vector space dimension, and therefore so do

$$
\left(\mathbb{C}\left[e_{1}, \ldots, e_{n}\right] / \operatorname{in}(J)\right) \otimes_{\text {Sym } g} \mathbb{C}_{\lambda} \text { and }\left(\mathbb{C}\left[e_{1}, \ldots, e_{n}\right] / J_{\Delta} \text { bc }\right) \otimes_{\text {Sym } g} \mathbb{C}_{\lambda} .
$$




\section{Nicholas Proudfoot}

This implies that $Q \otimes_{\text {Sym g }} \mathbb{C}_{\lambda}=0$. But since $Q$ is a submodule of a free module and $\lambda$ is generic, this implies that $Q=0$, and we are done.

Remark 5.2. In the last paragraph of the proof of Lemma 5.1, we invoked the statement that $\mathrm{H}^{\operatorname{dim} \mathfrak{M}}(\mathfrak{M})$ is (naturally) dual to $\mathrm{IH}_{T^{!}}^{*}\left(\mathfrak{N}^{!}\right) \otimes_{\text {Sym }} \mathbb{C}_{\lambda}$. This result, which is established in [BLPW12, 7.21], builds on an enormous amount of background material, and might be frustrating to a reader who does not want to take the time to learn all about hypertoric category $\mathcal{O}$ and symplectic duality. In fact, this was overkill; all we needed to know was that the dimension of $\mathrm{H}^{\operatorname{dim} \mathfrak{M}}(\mathfrak{M})$ is equal to that of $\operatorname{IH}_{T^{!}}^{*}\left(\mathfrak{N}^{!}\right) \otimes_{\text {Sym }} \mathbb{C}_{\lambda}$, which is the same as the total dimension of $\operatorname{IH}^{*}\left(\mathfrak{N}^{!}\right)$. This fact follows from [PW07, $3.5 \& 4.3$, which is considerably more accessible than [BLPW12, 7.21].

Corollary 5.3. The homology group $\operatorname{HP}_{0}(\mathscr{N})$ is a free Sym $\mathfrak{g}$-module with the same Hilbert series as $\mathrm{IH}_{T^{!}}^{*}\left(\mathfrak{N}^{!}\right)$.

Proof. This follows from Proposition 4.2 and Lemma 5.1 along with [PW07, 4.3] and [PS06, Proposition 1].

\section{The canonical isomorphism}

In this section we prove Theorem 3.1. Consider the hyperplane arrangement $\mathcal{H}=\left\{H_{1}, \ldots, H_{n}\right\}$ in the vector space $\mathfrak{g} \subset \mathfrak{t}^{n}$ where $H_{i}$ is the intersection of $\mathfrak{g}$ with the $i^{\text {th }}$ coordinate hyperplane of $\mathfrak{t}^{n} \cong \mathbb{C}^{n}$. (This is the hyperplane arrangement that is standardly associated with the hypertoric variety $\mathfrak{N}$ !, for example in [BD00] or [Pro08].) A flat of $\mathcal{H}$ is a subspace of $\mathfrak{g}$ obtained by intersecting some (possibly empty) subset of the hyperplanes. Let $L_{\mathcal{H}}$ be the poset of flats of $\mathcal{H}$, ordered by reverse inclusion.

The set $L_{\mathcal{H}}$ has a topology in which $U \subset L_{\mathcal{H}}$ is open if and only if whenever $F \leqslant F^{\prime}$ and $F^{\prime} \in U$, we have $F \in U$. If $\mathcal{S}$ is a sheaf on $L_{\mathcal{H}}$ and $F$ is a flat, let $\mathcal{S}(F)$ be the stalk of $\mathcal{S}$ at $F$. For each $F$, there is a minimal open set $U_{F}$ containing $F$, so $\mathcal{S}(F)$ is simply equal to $\mathcal{S}\left(U_{F}\right)$. We have $U_{F} \subset U_{F^{\prime}}$ if and only if $F \leqslant F^{\prime}$, therefore we have a collection of restriction maps $r\left(F, F^{\prime}\right): \mathcal{S}\left(F^{\prime}\right) \rightarrow \mathcal{S}(F)$ for every pair of comparable flats. By [BP09, 1.1], a sheaf is completely determined by its stalks and these restriction maps. Let $\mathcal{A}$ be the sheaf of algebras with $\mathcal{A}(F)=\operatorname{Sym}(\mathfrak{g} / F)$, along with the obvious restriction maps. This sheaf is called the structure sheaf of $L_{\mathcal{H}}$.

We will be interested in sheaves of graded $\mathcal{A}$-modules on $L_{\mathcal{H}}$. A sheaf $\mathcal{L}$ of graded $\mathcal{A}$-modules is called a minimal extension sheaf if it satisfies four properties:

$-\mathcal{L}$ is indecomposable;

$-\mathcal{L}$ is flabby;

- $\mathcal{L}(F)$ is a free $\mathcal{A}(F)$-module for all $F$;

$-\mathcal{L}(\mathfrak{g}) \cong \mathcal{A}(\mathfrak{g})=\mathbb{C}$.

Such a sheaf exists by [BP09, 1.10]. For any two minimal extension sheaves $\mathcal{L}$ and $\mathcal{L}^{\prime}$, there exists an isomorphism of $\mathcal{A}$-modules from $\mathcal{L}$ to $\mathcal{L}^{\prime}$, and this isomorphism is unique up to scalar multiplication [BP09, 2.7]. Furthermore, there is a particular minimal extension sheaf $\mathcal{L}$ (defined by applying a certain localization functor to the equivariant IC-sheaf of $\mathfrak{N}^{!}$) with the property that $\mathcal{L}(0)=\mathrm{IH}_{T^{!}}^{*}\left(\mathfrak{N}^{!}\right)[\mathrm{BP} 09,2.7]$.

To prove Theorem 3.1, we will find another minimal extension sheaf $\mathcal{M}$ with the property that $\mathcal{M}(0)$ is canonically isomorphic to $\mathrm{HP}_{0}(\mathscr{N})$. This will get us an isomorphism of modules over $\mathcal{A}(0)=$ Sym $\mathfrak{g}$ between $\mathcal{L}(0)=\mathrm{IH}_{T^{!}}^{*}\left(\mathfrak{N}^{!}\right)$and $\mathcal{M}(0) \cong \mathrm{HP}_{0}(\mathscr{N})$. Since the isomorphism between 


\section{Hypertoric Poisson homology in DEGReE Zero}

$\mathcal{L}$ and $\mathcal{M}$ is unique up to scalar multiplication, the isomorphism between stalks at 0 will be canonical up to scalar multiplication. It can then be made completely canonical by noting that both $\mathrm{IH}_{T^{!}}^{*}\left(\mathfrak{N}^{!}\right)$and $\mathrm{HP}_{0}(\mathscr{N})$ are canonically isomorphic to $\mathbb{C}$ in degree zero.

Recall that the data with which we began in Section 2 was an inclusion of tori $G \hookrightarrow T^{n}$, or equivalently an inclusion of abelian Lie algebras $\mathfrak{g} \rightarrow \mathfrak{t}^{n}$. For each flat $F$, let

$$
\mathfrak{t}_{F}^{n}:=\mathfrak{t}^{n} / \mathbb{C}\left\{e_{i} \mid F \not \subset H_{i}\right\},
$$

and consider the inclusion $\mathfrak{g} / F \hookrightarrow \mathfrak{t}_{F}^{n}$. With this starting point, we can repeat all of the constructions in this paper with $F$ in the subscript.

Define two sheaves of graded $\mathcal{A}$-modules $\mathcal{M}$ and $\mathcal{R}^{\text {bc }}$, where

$$
\mathcal{M}(F):=\operatorname{Sym}_{F}^{n} / J_{F} \quad \text { and } \quad \mathcal{R}^{\mathrm{bc}}(F):=\operatorname{Sym}_{F}^{n} / J_{\Delta_{F}^{\mathrm{bc}}},
$$

with the obvious restriction maps. Lemma 5.1 tells us that $\mathcal{M}$ admits a filtration whose associated graded module is isomorphic to $\mathcal{R}^{\mathrm{bc}}$. We know that $\mathcal{R}^{\mathrm{bc}}$ is a minimal extension sheaf by [BP09, 3.9], thus the same is true of $\mathcal{M}$. This completes the proof of Theorem 3.1.

Remark 6.1. We now summarize our approach to the proof of Theorem 3.1, in case the central idea got lost in the machinery of [BP09]. Two free graded Sym $\mathfrak{g}$-modules $L$ and $M$ with the same Hilbert series are necessarily isomorphic, but not canonically so. The problem is that graded Sym $\mathfrak{g}$-modules are very floppy objects - that is, they have lots of automorphisms.

On the other hand, a minimal extension sheaf on $L_{\mathcal{H}}$ is a rigid object - that is, it has only scalar automorphisms - whose space of global sections is a graded Sym $\mathfrak{g}$-module. Thus, if $L$ and $M$ can be promoted to minimal extension sheaves $\mathcal{L}$ and $\mathcal{M}$, then $\mathcal{L}$ and $\mathcal{M}$ are canonically isomorphic (up to scalars), which induces a canonical isomorphism between $L$ and $M$ (up to scalars). If $L$ and $M$ are both canonically isomorphic to $\mathbb{C}$ in degree zero, then we can do away with the scalar ambiguity.

This becomes particularly bizarre when $M$ admits a filtration such that gr $M$ is canonically isomorphic to $L$, and this lifts to a filtration on $\mathcal{M}$ such that $\operatorname{gr} \mathcal{M}$ is isomorphic to $\operatorname{gr} \mathcal{L}$. In this case, you don't really expect $L$ and $M$ to be canonically isomorphic, but rigidity of minimal extension sheaves tells you that they are.

In our case we have three modules, $L=\operatorname{IH}_{T^{!}}^{*}\left(\mathfrak{N}^{!}\right), R^{\mathrm{bc}}=\operatorname{Sym}^{n} / J_{\Delta^{\mathrm{bc}}}$, and $M=\operatorname{HP}_{0}(\mathscr{N})$, along with a filtration on $M$ with $\operatorname{gr} M \cong R^{\mathrm{bc}}$ (Lemma 5.1). The work of lifting $L$ and $R^{\mathrm{bc}}$ to sheaves $\mathcal{L}$ and $\mathcal{R}^{\mathrm{bc}}$ on $L_{\mathcal{H}}$ and proving that these sheaves are minimal extension sheaves is done in [BP09]. The work of lifting $M$ to a sheaf $\mathcal{M}$ on $L_{\mathcal{H}}$ is easy, and we prove that it is a minimal extension sheaf by lifting the filtration on $M$ to one on $\mathcal{M}$ with gr $\mathcal{M} \cong \mathcal{R}^{\text {bc }}$. This gives us canonical module isomorphisms $L \cong R^{\mathrm{bc}} \cong M$, which is exactly the statement of Theorem 3.1.

Finally, we note that we used a similar argument in [BP09] to show that $\mathrm{IH}_{T^{!}}^{*}\left(\mathfrak{N}^{!}\right)$is canonically isomorphic to the Orlik-Terao algebra of $\mathcal{H}$.

\section{ACKNOWLEDGEMENTS}

The author is grateful to Travis Schedler for introducing him to Poisson homology and explaining the various conjectures in [ES11]. Thanks are also due to Victor Ostrik and Tony Licata for help with tracking down intersection cohomology Poincare polynomials of nilpotent orbit closures and Uhlenbeck spaces, respectively. 


\section{Nicholas Proudfoot}

\section{REFERENCES}

AL98 J. Alev and T. Lambre, Comparaison de l'homologie de Hochschild et de l'homologie de Poisson pour une déformation des surfaces de Klein, Algebra and operator theory (Tashkent, 1997), Kluwer Acad. Publ., Dordrecht, 1998, 25-38.

Bar00 V. Baranovsky, Moduli of sheaves on surfaces and action of the oscillator algebra, J. Differential Geom. 55 (2000), no. 2, 193-227.

BD00 R. Bielawski and A. Dancer, The geometry and topology of toric hyperkähler manifolds, Comm. Anal. Geom. 8 (2000), no. 4, 727-760.

BLPW10 , Gale duality and Koszul duality, Adv. Math. 225 (2010), no. 4, 2002-2049. http: //dx.doi.org/10.1016/j.aim.2010.04.011

BLPW12 , Hypertoric category $\mathcal{O}$, Adv. Math. 231 (2012), no. 3-4, 1487-1545. http://dx.doi. org $/ 10.1016 / j$.aim.2012.06.019

BP09 T. Braden and N. Proudfoot, The hypertoric intersection cohomology ring, Invent. Math. 177 (2009), no. 2, 337-379. http://dx.doi.org/10.1007/s00222-009-0181-y

BPW12 T. Braden, N. Proudfoot, and B. Webster, Quantizations of conical symplectic resolutions I: local and global structure, arXiv:1208.3863 (2012).

ES10 P. Etingof and T. Schedler, Traces on finite $\mathcal{W}$-algebras, Transform. Groups 15 (2010), no. 4, 843-850. http://dx.doi.org/10.1007/s00031-010-9103-8

ES11 _ Poisson traces for symmetric powers of symplectic varieties, Int. Math. Res. Notices, to appear, arXiv:1109.4712. http://dx.doi.org/10.1093/imrn/rnt031

ES12 Z Zeroth Poisson homology of symmetric powers of isolated quasihomogeneous surface singularities, J. Reine Angew. Math. 667 (2012), 67-88. http://dx.doi.org/10.1515/CRELLE. 2011.124

MOV05 A. Malkin, V. Ostrik, and M. Vybornov, The minimal degeneration singularities in the affine Grassmannians, Duke Math. J. 126 (2005), no. 2, 233-249. http://dx.doi.org/10.1215/ S0012-7094-04-12622-3

Nak99 H. Nakajima, Lectures on Hilbert schemes of points on surfaces, University Lecture Series, vol. 18, American Mathematical Society, Providence, RI, 1999.

Nam10 Y. Namikawa, Poisson deformations of affine symplectic varieties, II, Kyoto J. Math. 50 (2010), no. 4, 727-752. http://dx.doi.org/10.1215/0023608X-2010-012

Nam11_ Poisson deformations of affine symplectic varieties, Duke Math. J. 156 (2011), no. 1, 51-85. http://dx.doi.org/10.1215/00127094-2010-066

Pro08 N. Proudfoot, A survey of hypertoric geometry and topology, Toric Topology, Contemp. Math. vol. 460, Amer. Math. Soc. Providence, RI, 2008, 323-338. http://dx.doi.org/10.1090/ conm/460/09027

PS06 N. Proudfoot and D. Speyer, A broken circuit ring, Beiträge Algebra Geom. 47 (2006), no. 1, 161-166.

PW07 N. Proudfoot and B. Webster, Intersection cohomology of hypertoric varieties, J. Algebraic Geom. 16 (2007), no. 1, 39-63. http://dx.doi.org/10.1090/S1056-3911-06-00448-6

Nicholas Proudfoot njp@uoregon.edu

Department of Mathematics, University of Oregon, Eugene, OR 97403, USA 